\section{Rol de cuidadores migrantes de mayores dependientes y opinión de la asistencia sanitaria en atención primaria}

\section{Role of migrant caregivers of dependent elderly and opinion of health care in Primary Care}

Remedios Rojano-Pérez'

M. ${ }^{a}$ Magdalena Cuevas-Fernández Gallego²

Lucinda Morales-Infante ${ }^{3}$

Pilar Izquierdo-Luzón ${ }^{4}$

Yolanda Carrión-Velasco ${ }^{5}$

Esperanza Martín-Salvador ${ }^{6}$

Juan Carlos Morilla-Herrera ${ }^{7}$

1. Doctora en Ciencias de la Salud, Facultad Ciencias de la Salud, Universidad de Málaga. Enfermera Gestora de Casos, C.S. Victoria, Distrito Sanitario Málaga-Guadalhorce, Málaga, España.

2. Doctora en Ciencias de la Salud, Facultad Ciencias de la Salud, Universidad de Málaga. Profesora asociada clínica, Universidad de Málaga (UMA). Enfermera Gestora de Casos, C.S. Portada alta, Málaga, España.

3. Enfermera Gestora de Casos. C.S. Torcal-San Andrés y C.S. Delicias. Málaga. España.

4. Enfermera Gestora de Casos. C.S. Alhaurín de la Torre. Alhaurín de la Torre. Málaga. España.

5. Enfermera Gestora de Casos. C.S. La Luz. Málaga. España.

6. Enfermera Gestora de Casos hospitalaria. Hospital Clínico Universitario Málaga Virgen de la Victoria. Málaga. España.

7. Doctor en Ciencias de la Salud, Facultad Ciencias de la Salud, Universidad de Málaga. Profesor asociado clínico UMA, Enfermero especialista en Geriatría, Coordinador Unidad de Residencia Málaga-Guadalhorce, Málaga, España.

*Autor para correspondencia.

Correo electrónico: remedios.rojano.sspa@juntadeandalucia.es (Remedios Rojano-Pérez).

Recibido el 18 de junio de 2019; aceptado el 7 de octubre de 2019.

\section{RESUMEN}

Objetivo: Describir el proceso de experiencias de vida de las personas migrantes que se convierten en cuidadores de mayores en situación de dependencia y conocer su opinión de la asistencia sanitaria. Diseño: Investigación cualitativa fenomenológica basada en la metodología de la teoría fundamentada. Realizado en el distrito sanitario Málaga-Guadalhorce durante 2017 y 2018. La captación se realizó mediante las enfermeras de familia de los centros de salud y a través de la base de datos de programas de atención domiciliaria de siete centros de salud. Se realizaron 17 entrevistas en profundidad a cuidadores migrantes que llevan cuidando un año o más a personas dependientes, hasta la saturación de la información. Resultados:

En este estudio se han descrito tres fases: fase inicial, la persona siente la necesidad de cambio y motivación para venir; segunda fase, búsqueda de trabajo en nuestro país y asunción de nuevas actividades; tercera fase, estrategia de afrontamiento e incertidumbre por expectativas de futuro. Manifiestan mejoras en salud en relación con: tiempos reducidos de consulta, dificultad para ver especialistas, falta de regularidad en las visitas médicas en domicilio y discriminación sufrida por ser migrante. Conclusión: Los migrantes traen experiencia en el cuidado de sus diferentes culturas que deben ser exploradas.

El refuerzo positivo de los empleadores, salarios y tiempo libre han sido condicionantes de mejora de satisfacción laboral y apoyo social. Lo mejor

valorado del servicio sanitario son las visitas domiciliarias por el refuerzo positivo. Al mismo tiempo se quejan de que no reciben todas las que necesitan.

Los profesionales deben realizar intervenciones en cada fase para facilitar la adopción de este rol, tratar de solucionar los problemas que surjan e informar a los organismos de salud de nuestra comunidad autónoma sobre dónde están los puntos débiles que deben mejorarse y de qué puntos fuertes partimos.

PALABRAS CLAVE: Cuidadores, migración humana, prestación de atención de salud.

\section{ABSTRACT}

Objective: To describe the process of life experiences of migrants who become caregivers of elderly people in situations of dependency and to know their opinion about health care. Design: Phenomenological qualitative research based on the methodology of grounded theory. Carried out in the Málaga-Guadalhorce health district, during 2017 and 2018. Recruitment was carried out by nurses from families in the Health Centers and through a database of home care programs, from seven health centers. 17 in-depth interviews were carried out with migrant caregivers, who have been caring for a year or more, dependent people, until the saturation of the information. Results: Three phases have been described in this study. In the initial stage the person feels the need for change and motivation to come; second stage of job search in our country and assume new activities; third stage of coping strategy and uncertainty for future expectations. They show improvements in health in relation to: reduced medical consultation time, it is difficult to consult with specialists, lack of regularity in home doctor visits and discrimination suffered by being a migrant. Conclusion: Migrants bring experience in caring for their different cultures that must be explored. The positive reinforcement of employers, salaries and free time have been conditions for improving job satisfaction and social support. What they value most in the health service are home visits for positive reinforcement, at the same time they complain that they do not receive all they need. Professionals should make interventions at each stage to facilitate the adoption of this role.

KEYWORDS: Caregivers, human migration, delivery of health care, qualitative research. 


\section{E INTRODUCCIÓN}

Varias estrategias han sido utilizadas para minimizar las consecuencias negativas en el cuidador familiar: apoyo formal, soporte emocional, prestaciones económicas, ayuda en los cuidados y liberar tiempo con centros de día y residencias ${ }^{1}$.

Son precisamente las cuidadoras migrantes el recurso mayoritario con el que se descarga parcialmente a las familias del cuidado de sus mayores. Por otro lado, hay que tener en cuenta a las personas migrantes que proporcionan el cuidado en los domicilios. El perfil formal de los cuidadores migrantes no profesionales suele ser: mujer, joven, sana, con estudios secundarios y/o superiores ${ }^{2}$, con iniciativas y ganas de mejorar sus vidas y la de sus familias. Es importante resaltar que el proceso migratorio deriva en un trabajo personal intenso de adaptación y duelo que implica dejar atrás familia, amigos y cultura. Es también una experiencia enriquecedora que hace madurar, ser más independiente, aumenta la capacidad crítica y la capacidad de elegir desde los conocimientos adquiridos de las diferentes culturas visitadas y los diferentes idiomas aprendidos ${ }^{3}$.

España es un país de Europa donde es fácil acceder a un empleo de cuidador domiciliario, con un importante sector de economía sumergi$\mathrm{da}$, fundamental para encontrar un trabajo en el comienzo del ciclo migratorio. Las ventajas que ofrece este recurso en cuanto a disponibilidad horaria (mediante la modalidad de interna, que es una cuidadora que atiende las necesidades del mayor durante 24 horas del día, en muchas ocasiones con un sueldo precario, y sobre todo en las recién llegadas) y condiciones laborales, que favorece a los empleadores ${ }^{1}$, son dos aspectos que inciden en su demanda junto con las deficiencias del sistema público de protección social y determinante para que un colectivo vulnerable se incorpore a esta actividad. A ello se une el deseo de las familias, de las personas cuidadas y desde las instituciones al conceder más importancia a los cuidados de larga duración en el hogar que a los cuidados prestados en las instituciones ${ }^{4}$.

Por todo lo expuesto, el apoyo formal por cuidadores migrantes es el más utilizado por las familias españolas y este fenómeno debe ser estimado por los sistemas de salud para adaptar la atención a su propia salud y a la provisión de cuidados que realizan 5 .

Mediante un enfoque transcultural, se pretende describir el perfil y conocer cómo perciben los cuidadores migrantes su carga de trabajo y el apoyo social percibido, y los conocimientos y las vivencias como cuidadores de personas dependientes desde sus respectivas culturas. Es preciso incorporar la opinión de los cuidadores inmigrantes en la organización de la asistencia facilitando y conciliando los componentes culturales de su trabajo diario con nuestros criterios de cuidados a personas dependientes.

Los objetivos de la investigación son:

- Explorar los significados de las experiencias de vida como cuidadores en un país diferente.

- Conocer la opinión de personas migrantes como cuidadores de la asistencia sanitaria andaluza.

\section{- PARTICIPANTES Y MÉTODO}

\section{Diseño}

Investigación cualitativa fenomenológica basada en teoría fundamentada y desarrollada mediante entrevistas en profundidad a cuidadores formales migrantes de mayores dependientes durante el año 2018 en los centros de salud Alameda-Perchel, Victoria, San Andrés-Torcal, Delicias y Puerta blanca del Distrito Sanitario Málaga.
El guion inicial de las entrevistas se estructuró a partir de las siguientes dimensiones: experiencia de vida en sus países de origen y en España, cultura del cuidado, satisfacción laboral, red de apoyo social y percepción del sistema de salud. Las entrevistas fueron grabadas en audio y transcritas literalmente para su análisis posterior.

\section{Muestra y participantes}

Las personas cuidadoras fueron localizadas a través de visitas domiciliarias de enfermería a personas en situación de dependencia y a través de base de datos de programas de atención domiciliaria. Se usaron como criterios de inclusión: mujeres y hombres cuidadores migrantes, mayores de 18 años y con un año o más cuidando a personas dependientes.

Se llevó a cabo un muestreo no probabilístico teórico e intencional, de acuerdo con los principios de la teoría fundamentada, que buscó analizar casos significativos para la comprensión del problema hasta conseguir saturación de la información. Cuarenta y dos cuidadores fueron invitados a participar; veintitrés se negaron y dos habían participado en la prueba-piloto. La muestra fue ampliándose a partir del preanálisis, hasta alcanzar la saturación. En total se necesitó entrevistar a 17 personas cuidadoras migrantes. El ámbito situacional de la muestra lo conformaron dos centros de salud con poder adquisitivo medio-bajo y tres centros de salud que tienen presencia de núcleos urbanos con poder adquisitivo medio-bajo y medio-alto, lo que facilita la representatividad de la información.

Se informó a los participantes sobre la grabación de las entrevistas, finalidad y procedimiento del estudio, así como sobre las garantías de confidencialidad y anonimato (fig. 1).

Las entrevistas en profundidad se realizaron en el centro de salud. Se generó un guion de entrevistas a partir de los bloques temáticos necesarios para alcanzar el objetivo propuesto. Así mismo, se realizaron preguntas abiertas para facilitar a los entrevistados expresarse libremente y se exploraron aquellos temas emergentes relevantes para los objetivos propuestos. Las entrevistas fueron transcritas literalmente para construir los documentos primarios. El material grabado, las transcripciones y los documentos de consentimiento fueron conve-

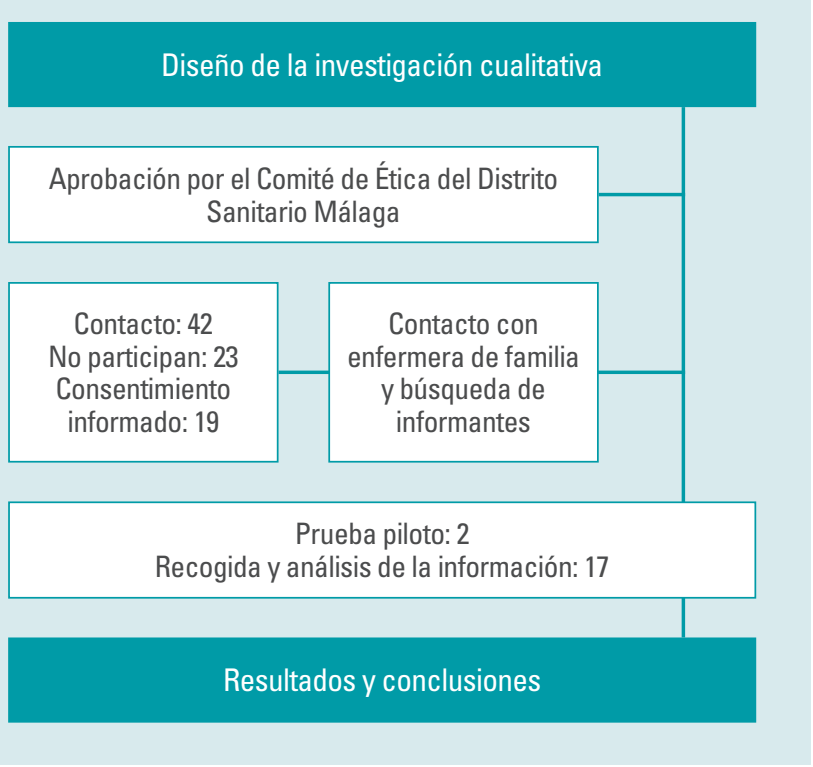

Figura 1. Esquema del estudio. 
nientemente archivados para poder identificar a la investigadora que realiza la entrevista, el centro de salud y la fecha. Se han cumplido las normas éticas del Comité de Investigación y de la Declaración de Helsinki de 1975 con la revisión de octubre del 2000, disponible en http://www.wma.net/s/policy/b3.htm.

\section{Análisis}

El análisis de la información se realizó según el enfoque de la teoría fundamentada propuesto por Glaser at al. ${ }^{6}$. En primer lugar, se llevó a cabo un microanálisis de la información con una codificación abierta, seguido de la codificación selectiva, para integrar categorías. Para realizar la codificación teórica y establecer las conexiones entre categorías se utilizó el método comparativo constante, con la identificación de similitudes y diferencias. Se analizaron las concurrencias surgidas del análisis. En todo el proceso de codificación y de categorización de la información se usaron memos, que contribuyeron a la codificación teórica.

Para el proceso de análisis se ha utilizado el software Atlas Ti 7 y la codificación se realizó por parte de dos investigadores diferentes para triangular la información y aumentar la credibilidad del estudio ${ }^{7}$.

\section{- RESULTADOS Y DISCUSIÓN}

Se realizaron 17 entrevistas a informantes-clave: 2 hombres y 15 mujeres, de edades comprendidas entre los 22 y los 51 años.

Los dos hombres y la mayoría de las mujeres eran de procedencia latinoamericana, y 4 mujeres provenían de países del Este.

Respecto al nivel de estudios de las personas entrevistadas: cuatro tienen estudios primarios, nueve secundarios y cuatro son universitarios.

Respecto a su red familiar: todos tienen hijos en sus países de origen menos una participante que es soltera sin hijos y tres cuidadores que tienen además pareja e hijos en España.

El idioma predominante es el español, seguido del ruso, el rumano y el ucraniano.

De las 17 personas entrevistadas, 8 de las mujeres trabajan internas como cuidadoras. El salario medio que cobran es de 600 euros.

Los resultados del perfil sociodemográfico de los entrevistados coinciden con diferentes estudios ${ }^{1,2}$; pensamos que esto se debe a que son estudios dentro de nuestro ámbito nacional: la mayoría de los cuidadores entrevistados son latinoamericanos que comparten el idioma español, eliminando así la barrera del idioma para los migrantes. En relación con el cuidado, tradicionalmente en todas las culturas este está vinculado a la mujer; ello explicaría el predominio del género femenino en el cuidado a mayores que es el que nos compete en este estudio.

Los discursos coinciden en su mayor parte, lo que nos permite presentar los resultados integrando todas las técnicas utilizadas, descritas en el análisis. Se reafirman estos resultados en las propias palabras de las personas entrevistadas.

El proceso que lleva a una persona a abandonar su país para lograr su proyecto de vida pasa por diferentes fases:

1. Fase inicial: la persona siente la necesidad de mejorar y eso le motiva a migrar, aunque en ocasiones la necesidad de conocer otras culturas también supone un aliciente.

2. Fase media, que comprende el proceso de búsqueda de trabajo en nuestro país en el que deben asumir nuevas actividades imprevistas.

3. Fase final, de adaptación a la nueva situación poniendo en marcha estrategias de afrontamiento cognitivo que acompañan a la incertidumbre sobre sus expectativas de futuro.

\section{La motivación para venir a nuestro país}

Los cuidadores entrevistados reconocen realizar trabajos precarios con sueldos mínimos, que no les permite sacar a sus familias adelante dignamente en su país de origen. Su objetivo en la gran mayoría es trabajar y enviar dinero a casa, para el cuidado y estudios de sus hijos. Se plantearon salir de sus respectivos países por sus desigualdades sociales y de género, y en ocasiones quieren dejar atrás separaciones traumáticas de sus parejas. Estos resultados coinciden con el estudio de Valera ${ }^{8}$. Se encuentran con un trabajo de economía sumergida que por un lado les facilita el acceso y por otro les impone salarios austeros en destino, pero que les permite realizar sus objetivos en sus países de origen: los logros de estos objetivos, los motiva y explican su migración. No quedan exentos de sufrir los pormenores del proceso migratorio 9 (tabla 1 ).

\section{Proceso migratorio}

Uno de los fenómenos más desestabilizadores de la vida de las personas es la migración. No obstante, señalan algunos factores positivos del proceso migratorio: la experiencia personal que les permite ser independientes aumenta su capacidad crítica y de toma de decisiones desde los conocimientos adquiridos y también al "beber" de las diferentes culturas con las que conviven en el país de acogida.

En los relatos de su experiencia de vida, las personas entrevistadas buscan también independencia personal, social y económica. Así lo relatan en sus discursos muchas de las mujeres migrantes (tabla 1). El proyecto migratorio es una oportunidad de realización personal, manifestada en el deseo de comprar una vivienda en origen para vivir con sus hijos, hasta en la terminación de sus propios estudios.

Inevitablemente, el proceso migratorio deriva en un trabajo personal intenso de adaptación y duelo. Esto explica que cada proceso migratorio es único. El logro de los objetivos se verá influenciado por características personales, condiciones de la migración, país de destino, apoyo social, nacionalidad, edad, conocimiento del idioma, etc. ${ }^{10}$ (tabla 1 ).

\section{Adaptación a la nueva situación}

Las personas entrevistadas expresan que no todo es fácil en sus vidas, pues se enfrentan al trabajo como internas con sueldos precarios, la falta de contrato, las múltiples tareas. Además de sentir a sus seres queridos lejos (tabla 1).

Pensamos que, en los países de acogida, las personas recién llegadas interactúan en función de su situación laboral o de la localización de su vivienda, así como de su participación en asociaciones que defienden los derechos de los trabajadores o de los vecinos de sus barrios: esos son los puntos de partida de vínculos de solidaridad y de amistad. Al mismo tiempo forman parte de asociaciones constituidas por miembros de sus pueblos, con miras a mejorar sus condiciones de vida. Todos estos factores influyen y explican que unas personas se integren mejor que otras en el país de acogida. También se preocupan por hacer resaltar social y políticamente sus especificidades culturales.

\section{Expectativa de futuro}

La gran mayoría quiere volver a su país de origen tras cumplir su proyecto de vida de mejora económica y social para ellos mismos y sus familias (tabla 1).

Los entrevistados ponen de manifiesto que el permiso de residencia y los efectos beneficiosos de un trabajo remunerado aumentaron el bienes- 
Tabla 1. Proceso migratorio y vivencias

\begin{tabular}{|c|c|}
\hline Categoría & Discursos de los participantes \\
\hline $\begin{array}{l}\text { Motivación } \\
\text { para venir }\end{array}$ & $\begin{array}{l}\text { "La motivación más grande es poder darles a mis hijos cosas que nosotros no tuvimos, estudios....". E } 14 \\
\text { "No tenía grandes cosas, pero vivía bien. Hasta que vino la crisis y tuvimos que migrar en cuestión de un año". E } 8 \\
\text { "Para ver cómo viven en otros países. Quiero aprender cocina española". E } 4 \\
\text { "Hace } 10 \text { años, más o menos, yo pensaba que era la mujer maravillas. Seguro que iba a cambiar a mi marido, mi niña nació y no cambió. } \\
\text { Seguía de mujeriego, de pegador, me vine". E } 12 \\
\text { "Todo muy caro o al mismo nivel como en España, es socialista y los precios capitalistas y no se puede vivir". E } 7\end{array}$ \\
\hline $\begin{array}{l}\text { Proceso } \\
\text { migratorio }\end{array}$ & $\begin{array}{l}\text { "He conseguido pagar en casi } 9 \text { meses mi deuda para venir. Me dolió dejarle a mi familia, la distancia es dura”. E } 6 \\
\text { "Pero yo aprendí muchas cosas, a ser más madura. Muchas cosas me costaron a mí decidir para venir. Y ahora sé lo que es estar lejos". E } 12\end{array}$ \\
\hline $\begin{array}{l}\text { Adaptación a la } \\
\text { nueva situación }\end{array}$ & $\begin{array}{l}\text { "Yo por ejemplo me adapto a las costumbres de este país. Porque yo soy una migrante y tengo que adaptarme". E } 1 \\
\text { "Me llevo muy bien con toda la gente de aquí, pero... tanto como feliz, tampoco, no por el hecho del trabajo. Es por más que muchas veces } \\
\text { uno quiera sacarse del lado los problemas que tiene encima, no puedes". E14 }\end{array}$ \\
\hline $\begin{array}{l}\text { Expectativas } \\
\text { de futuro }\end{array}$ & $\begin{array}{l}\text { "Me preocupa el futuro, me hago grande y acá no tengo mucho tiempo trabajado con papeles". "Se gana muy poco, me preocupa por mi } \\
\text { familia y por mí". E } 17 \\
\text { "Con mi marido, ya vinimos juntos, y estamos los dos trabajando y queremos irnos este año". E } 13 \\
\text { "Me vine para poder comprar una casa y vivir allí con mis hijos. Mi hermana cuando se murió mi madre vendió mi casa que yo le compré a } \\
\text { mi madre, para que estuviera con mis hijos. Y tuve que empezar de nuevo, pero esta vez a nombre de mis hijos y mío". E } 12\end{array}$ \\
\hline $\begin{array}{l}\text { Vivencias en } \\
\text { país de acogida }\end{array}$ & $\begin{array}{l}\text { "Trabajo de interna, ahora que llevo } 2 \text { años me han subido un poquito, pero he estado cobrando 600, sin papeles. Me dolía la espalda. } \\
\text { Se aguanta mucho, por los papeles". E } 1 \\
\text { "Tengo que volver a estudiar para estar a nivel de los profesores de aquí, para nivelar mi carrera con ellos. Y por mis papeles". E } 9\end{array}$ \\
\hline
\end{tabular}

Origen: Entrevistas en profundidad.

tar psicológico (tabla 1), resultados que coinciden con el estudio de análisis de género desde la enfermería en cuidadoras migrantes de personas mayores ${ }^{11}$. Es indiscutible que la regularidad de su estatus de residencia (elimina la incertidumbre de ocultación), la estabilidad laboral y salarios competentes aumenta el bienestar psicológico.

La incertidumbre y el malestar psicológico se explican por diferentes factores: cuando se quedan sin trabajo, sin regularización y sin tener el dinero suficiente para lograr sus objetivos. Y en los que se quedan también preocupa la jubilación y el bienestar de sus familias y el suyo propio como bien referencia un cuidador en su discurso (tabla 1).

\section{Experiencia de vida en España}

En sus relatos manifiestan que el país de acogida les facilita la oportunidad de cumplir sus objetivos personales y familiares, siendo ello su principal motivo de migración. También sufren en ocasiones por sus familias, debido a que surgen conflictos y negociaciones, producto de los cuales no siempre se toman decisiones racionalmente óptimas (tabla 1).

Objetivamos en el presente estudio y en el de Oliva ${ }^{10}$ que en Espańa la mayoría empieza trabajando como interna, lo que facilita el acceso a una vivienda al tiempo que disminuye significativamente los gastos en alimentación, transporte y otros artículos de uso personal, permitiendo cierto ahorro y con él la posibilidad de cumplir con las deudas contraídas de ayuda económica a la familia en origen a través de las remesas. Consideramos que este tipo de empleo conlleva, a la larga, pérdida de intimidad y falta de descanso que difícilmente permitirá mejorar sus condiciones laborales o conseguir otro empleo mejor. Además, sus oportunidades de trabajo son de baja cualificación y no siempre se correlaciona con el nivel de estudios que poseen ${ }^{12}$.

\section{Cultura del cuidado}

Las personas que vienen a nuestro país ya traen una cultura del cuidado de sus respectivos países, son cuidadoras de sus abuelos, padres y suegros: a menudo conviven con ellos. No tenemos en cuenta este conocimiento, que puede ser un potencial del cual partir para dar cuidados de calidad a nuestros mayores dependientes, además de facilitar una formación acreditada impartida por profesionales que empodere a las personas cuidadoras para administrar adecuadamente estos cuidados.

Las personas entrevistadas nos informan de que son generalmente los familiares de las personas cuidadas quienes enseñan a los cuidadores respecto al cuidado de necesidades básicas. En relación con las curas y adherencia al tratamiento, son las enfermeras en domicilio las que enseñan. Las personas que administran los cuidados a nuestros mayores realizan limpieza de la casa, cocinan, planchan, compran, los acompañan al médico, pasean con ellos, realizan la vigilancia nocturna, el aseo personal, administran tratamiento, procuran el control de la tensión arterial, glucemias capilares, dan alimentación por sonda nasogástrica y realizan curas sencillas (tabla 2). Estos hallazgos son avalados en una reciente revisión sistemática ${ }^{13}$.

Consideramos que la realización de todas estas tareas tiene como consecuencia una carga de responsabilidad e incertidumbre por no disponer de conocimientos suficientes de riesgos, signos de alarma y actuaciones adecuadas y el consecuente aumento de exacerbaciones y/o ingresos hospitalarios por complicaciones de su cronicidad ${ }^{14,15}$.

Las personas entrevistadas describieron síntomas como tristeza, cansancio, ansiedad, alteraciones del sueńo y dolores de espalda relacionado con el grado de dependencia de la persona cuidada (tabla 2).

Las entrevistadas señalan el trabajo que supone tener bajo su responsabilidad la vida de una persona a la que es necesario atender, no úni- 
camente desde el punto de vista físico, también asistencial y emocional (tabla 2). Entendemos que, como consecuencia, las personas entrevistadas sienten una gran presión emocional.

También relataron consecuencias positivas del trabajo de cuidar, percibiendo el proceso de cuidados como una ayuda en la continuidad de sus proyectos que llena los vacíos emocionales ocasionados por la distancia con sus familias (tabla 2).

A pesar del habitual aislamiento que caracteriza esta actividad en el ámbito doméstico familiar, las personas entrevistadas interactuaron con personas con historias de vida, visiones y necesidades de cuidado diferentes (tabla 2), y consideramos que esto posibilitó a los cuidadores una apertura mental que impactó positivamente en sus propias historias de vida y de relación con sus familiares en el país de origen.

Según nuestros resultados, en la primera fase del cuidado deberían adoptarse intervenciones precoces y genéricas de tipo informativo, documentadas en soporte papel e internet, ya que todos manejan internet para comunicarse con sus familias en origen. Esta información puede darse en talleres de cuidadores, para cuidadoras de inicio, o en las visitas domiciliarias de las enfermeras de manera práctica y documentada, que disminuyan la incertidumbre a los cambios que se puedan producir. En la fase de adaptación al nuevo rol, las intervenciones deben ser personalizadas y focalizadas en problemas concretos de adaptación.

\section{Satisfacción laboral}

El análisis realizado ha permitido identificar que el cuidado realizado por las personas entrevistadas aporta importantes beneficios, tanto para quienes lo realizan como para quienes lo reciben, al no encontrarse solas estas últimas en muchos casos, y descarga en gran medida a las familias del cuidado (tabla 2).

El aspecto emocional del trabajo de cuidado fue el que más desafía y tensiona a las trabajadoras (tabla 2). Al tiempo que les recompensa afectivamente, les dificulta exigir sus derechos, lo que reproduce relaciones de explotación que atentan contra la revalorización del trabajo de cuidado y de quienes lo realizan ${ }^{13}$. En sus discursos, todos coinciden en la precariedad laboral, con sueldos bajos y un sinfín de tareas domésticas añadidas al cuidado de las personas dependientes, en su gran mayoría sin contrato (tabla 2). Todo sucede por una falta de regulación en el sector de cuidados y servicio doméstico, que agrava la situación en el caso de mujeres migrantes en situación irregular ${ }^{16}$.

La satisfacción laboral es valorada positivamente como se refleja en las entrevistas (tabla 2), pues les permite lograr un conjunto de competencias técnico-profesionales. Consideramos que deben ser certificadas de modo que les permita elegir la inserción en ámbitos institucionalizados del cuidado, o seguir en el sector doméstico y/o elegir seguir cuidando en domicilios.

Tabla 2. Cultura del cuidado y satisfacción laboral

\section{Categoría}

\begin{tabular}{l} 
Conocimiento cuidado \\
de sus culturas \\
\hline Cuidados realizados \\
en domicilio \\
\hline $\begin{array}{l}\text { Consecuencias } \\
\text { negativas } \\
\text { del cuidado }\end{array}$ \\
\hline Competencias técnicos \\
profesionales \\
\hline Consecuencias positivas \\
del cuidado
\end{tabular}

Relaciones emocionales y de explotación

Refuerzo positivo de los empleadores

\section{Discursos de los participantes}

"Era azafata de tren en mí país, ahí aprendí como estar en parto, un accidente y ayudar gente. Para cuidar lo llevo desde mi casa, vivía con mi abuela". E 4

"De la tensión tenía conocimientos. Porque trabajé en una farmacia. Pero de la insulina nada". E 6

"Yo tenía 16 años, vivía con mi abuela, yo cortaba uñas, ayudaba a vestir, peinaba...". E 7

"Cuidamos a los padres y en una parte también nos esclavizamos, porque haces tu vida de casada, pero la responsabilidad queda contigo y también el esposo te vuelca la suegra". E 12

"A las nueve limpio y desayuno con el abuelo, cambio las sábanas, limpio la casa. Cocino, plancho, mientras es la hora de almorzar. Preparo al abuelo para la siesta. A la noche, vuelvo a cambiar. Muchas veces no tengo tiempo para mí, a veces una persona se siente triste". E 7

"Le quito la venda para limpiarlo con la esponja húmeda, me enseñó la enfermera". E 5

"Le doy la comida por sonda, me enseño la hija". E 15

"Aprendido a mover con grúa. Me enseñó la enfermera de enlace". E 16

"Me enfermé de corazón y tensión, de tanto estrés, pero no podía dejar de trabajar, mi marido quedo parao". E 10

"La espalda es lo que más duele. También comer y estar sentada, me gané doce kilos, aunque salgo a andar. No puedo dormir, es por mí misma. No consigo dormir, al echarme a la cama". E 1

"Yo quería hacer cursos para cuidar ancianos, ahora que tengo experiencia, porque necesito capacitarme para trabajar en una residencia o en otro sitio que paguen mejor". E 3

"Ahora que ya tengo experiencias con las personas mayores, me gustaría estudiar enfermería". E 2

"Yo me siento muy bien cuidando a personas mayores. Yo no sé si es por el hecho de estar lejos de mi familia, una no tiene donde derrochar cariño. Una tiene hijos, le hace falta de mimar, hablándonos, dándonos un abrazo, son pequeñas cosas pero que nos hacen bien". E 5

"Me pagaba muy poquito, fue mi primer trabajo de interna. Cuando se enfermó él, me llamo a mí. Él no quería que nadie lo cuidara, más que yo". E 11

"Sí. Nosotras las dos solas estamos en la casa. La nuera también me llevo bien y con los nietos. Yo me siento como si fuera de la familia. Y ellos me tienen así". E 5

Origen: Entrevistas en profundidad. 
En nuestro estudio, la satisfacción laboral correlaciona positivamente con el bienestar percibido por los cuidadores, que reciben refuerzos positivos de sus empleadores (tabla 2). También se aprecia una correlación de la satisfacción laboral con los mejores salarios percibidos, coincidiendo con diferentes estudios ${ }^{17}$. Entendemos que el bienestar psicológico aumenta con el refuerzo positivo de los empleadores; esto pude ser explicado sin duda por el logro de recibir el reconocimiento del trabajo bien hecho y a su vez verse apoyados por las familias con las que trabajan. No podemos olvidar que la gran mayoría están solos en un país extranjero. Indiscutiblemente, en relación con la segunda correlación, cuanto mejores son los salarios, más satisfechos están los trabajadores.

\section{Red de apoyo social}

La población estudiada tiene en su mayoría amigos migrantes, participan con su iglesia ${ }^{18}$, asociaciones de migrantes y vecinos. Ellas mismas expresan la estabilidad emocional y el bienestar percibido que les aporta el poder hablar de sus problemas y compartir momentos de ocio con la comunidad del barrio (tabla 3). Conceptuamos que estos puntos de congregación laicos y de credos aumentan sus relaciones sociales y de apoyo.

En relación con la reagrupación familiar, en nuestro trabajo encontramos tres familias con hijos unos nacidos en el país de origen y otros en el de acogida, todos escolarizados en España, que expresaron su deseo de quedarse y tener ya su vida realizada aquí. En los tres casos trabajan ambos progenitores. Notifican llevar bien la convivencia entre ambas culturas, existiendo pues una transculturación equilibrada (tabla 3).

Las tres familias son latinoamericanas; pensamos que esto explica la transculturación por la similitud de las dos culturas, idioma ${ }^{18}$, la receptividad de la cultura de acogida, la edad de la persona. Consideramos que el tener sus hijos escolarizados en el país de acogida facilita su adap- tación. También discutimos que las características psicológicas pueden influir en el proceso de adaptación; por ejemplo: la personalidad o la voluntad de migrar y la posición socioeconómica.

Respecto a la entrevista con la cuidadora más joven, esta sabía que trabajaría en el servicio doméstico de cuidados, pero esperaba tener tiempo para sus estudios o proyecto de vida (tabla 3). Al entrar como interna, le resulta frustrante; esto coincide con el estudio de Bover ${ }^{18}$. Pensamos que en el caso de las más jóvenes, el disponer de poco tiempo libre o ninguno como interna y llegar con expectativas de trabajo en las profesiones en las que se han preparado en sus respectivos países de origen y/o continuidad de sus estudios las decepciona, al optar solo al mercado de trabajo del sector cuidados, al menos al principio mientras homologan sus títulos.

El apoyo social correlacionó positivamente con amigos espańoles, refuerzos positivos de los empleadores, con bienestar percibido y tiempo libre, resultados que coinciden con los encontrados en la revisión sistemática de los factores asociados a la presencia de malestar psicológico en poblaciones migrantes en $2017^{19}$. Suponemos que la correlación entre apoyo social y bienestar percibido por la persona, refuerzo positivo de los empleadores y amigos españoles puede explicarse por la mejor integración en el país de acogida y la satisfacción que aporta el reconocimiento del trabajo realizado, pero realmente al ser un hallazgo nuevo no podemos constatarlo categóricamente, necesitaríamos más entrevistas. Indiscutiblemente, la persona que dispone de tiempo libre aumenta sus posibilidades de relacionarse con los demás y por tanto mejora apoyo social.

\section{Percepción sobre equipo de salud y organización de la asistencia sanitaria}

En relación con los hospitales, las personas entrevistadas hablan de una notable mejora en relación con sus países de origen, en cuanto a infraes-

Tabla 3. Red de apoyo social. Percepción equipo de salud

\section{Categoría}

Redes de amigos
y ocio
Áreas de mejoras
en servicios
de salud

Discriminación en salud

Refuerzo positivo del equipo de salud
Discursos de los participantes

"Voy a nuestra iglesia. Tengo una amiga, está siempre ahí. Todos los días hablo con mis niños". E 6

"Estoy aquí con mi marido y mis hijos que vinieron chiquitos. Hace dos años con una amiga española estamos en un grupo de teatro del barrio. Me divierto mucho y se olvidan los problemas ese ratito". E 8

"Con mi marido, los domingos vamos a nuestra iglesia". E 13

"Yo tengo 22 años. Sabía que venía a cuidar viejitos, pero yo hice mi presentía de contabilidad y estuve trabajando en prácticas en empresas, me pagaban muy poco y por eso me vine y mis hermanas que están aquí, me llamaban. Pero llevo un año de interna y no puedo hacer nada de mis estudios. Tengo amigas de mi país. Tengo mucha añoranza de mi familia. Estoy regular de ánimos". E 15

"Comienzas hablar al médico y esos cinco minutos prácticamente ya. Parece que te escuchan, pero sin mirar. Hay mucha gente esperando, si tardas te miran mal. Con médicos o enfermeras bien. Pero yo no vi ningún médico en domicilios". E 3

"No, me gustó porque comparando con allá, si quiero, voy al ginecólogo, si quiero voy a la vista". E 8

"Las enfermeras hay que son más amorosas, otra que es más fría". E 15

"Una doctora me tocó, yo le hablaba y parecía que ella tenía problemas con gente migrante, me trataba muy mal". E 11

"Hace como seis meses me caí y me torcí la muñeca, y cuando yo fui al centro salud, no me quisieron atender, no tenía papeles, me puse a \|lorar. Y una señora que estaba en la cola me cogió de la mano y dijo: dame la hoja de reclamaciones. Y me atendió una doctora". E 10

“Sí, la enfermera buenísima. Yo le explico cómo lo estoy haciendo y ella me dice. Mi doctora, me sentí tan querida, para cuando estaba hundida, ella me levantó". E 1

"Llegó la enfermera a la casa hizo la prueba, ya me explicó, porque no sabía nada y muy bien". E 2

"Llamo enfermera, ella siempre enseña, muy bien, muy cariñosa". E 16 
tructura y existencia de material. También en la cercanía de los centros de salud de atención primaria a los domicilios (tabla 3).

Relatan la dificultad que encuentran para que los médicos de atención primaria los deriven a especialistas: tener que esperar varios días para que los vea su médico de familia y posteriormente varios meses para que los atienda el especialista, si el médico de familia así lo decide. También el poco tiempo que tienen en la consulta para explicar qué les sucede (tabla 3).

Las personas entrevistadas refieren que las enfermeras les enseñaron en el domicilio, realizaron educación sanitaria en relación con enfermedades crónicas, higiene, movilización del enfermo encamado; cuidados e higiene de sondas vesicales, nasogástricas, de estomas y catéteres de nefrectomía, enseñanza de curas superficiales, valorando positivamente este refuerzo (tabla 3).

En general, la mayoría tiene buenas experiencias con médicos de familia y enfermeras. Comentan las personas entrevistadas que les cuesta más ver a los médicos en domicilio (tabla 3 ).

Comentan también la discriminación que a veces sufren por el personal administrativo o sanitario que los recibe y los comentarios en la sala de espera de algunas de las personas autóctonas (tabla 3), coincidiendo con el estudio de EE. UU. de $2017^{20}$.

De los resultados se desprende que en la percepción del equipo de salud presentamos áreas en las que funcionamos bien (hospitales, infraestructuras, cercanías de los centros de atención primaria y valoración positiva de los refuerzos de educación para la salud en domicilios), así como áreas de mejora (demora para las citas de médicos de familia y especialistas, tiempos de atención en consulta médica). Consideramos, respecto a la discriminación por parte de los profesionales, que deberíamos realizar más formación en migración e igualdad y derechos humanos, en relación con la población en general desde los medios de comunicación, y desde la opinión publica deberíamos ser mas sensibles con estos temas y tratarlos desde el punto de vista de la multiculturalidad, ya que vivimos en un mundo global.

\section{- CONCLUSIÓN}

El estudio nos revela que los cuidadores migrantes traen experiencia en el cuidado de sus diferentes culturas que deben ser exploradas. El refuerzo positivo de los empleadores, salarios y tiempo libre han sido condicionantes de mejora de satisfacción laboral y apoyo social; un mercado laboral reglado para estos trabajadores del cuidado domiciliario mejora su satisfacción laboral y repercute en el trabajo realizado. Lo mejor valorado del servicio sanitario son las visitas domiciliarias por el refuerzo positivo; al mismo tiempo se quejan de que no reciben todas las que necesitan. Valoran la instituciones y cercanía de los centros a los domicilios y solicitan mejoras en los tiempos de atención en consulta médicas y meses de espera para especialistas.

\section{- LO CONOCIDO SOBRE EL TEMA}

Las personas cuidadoras migrantes son un soporte básico en el cuidado de nuestros mayores dependientes, proporcionan un bienestar a las familias de estos, al descargarlos o compartir el cuidado de sus seres queridos. La aceptación de este rol de inmediato supone para la persona cuidadora numerosos cambios en su vida. Son escasos los estudios que abordan la percepción de las personas cuidadoras migrantes sobre el equipo de salud que los atiende a ellos y a las personas cuidadas en domicilios.

\section{- QUÉ APORTA ESTE ESTUDIO}

Este estudio proporciona una mejor comprensión de la experiencia de convertirse en persona cuidadora proveniente de otro país y cultura. Traen cultura de cuidados desde sus respectivos países. Este trabajo empieza desde que asumen nuevas actividades de cuidado en las que emergen nuevas necesidades y consecuencias del propio cuidado, pasando por la adaptación a la nueva situación, hasta la incertidumbre de su expectativa de futuro. Los profesionales deben realizar intervenciones en cada fase para facilitar la adopción de este rol y tratar de solucionar los problemas que se le planteen. Valoran positivamente las visitas domiciliarias, con refuerzo positivo del cuidado. Los gobiernos deben regularizar el mercado de trabajo del cuidado a mayores dependientes en nuestro país.

Pone de manifiesto las líneas de mejoras en salud:

- Tiempos reducidos de atención en consulta médica.

- Dificultad de acceso a especialistas.

- Falta de regularidad en visitas médicas en domicilios.

- Discriminación por personal sanitario, no sanitario y personas autóctonas en los centros asistenciales

Conflicto de intereses

Los autores declaran no tener ningún conflicto de intereses.

\section{BIBLIOGRAFÍA}

1. Zueras P, Spijker J, Blanes A. Evolución del perfil de los cuidadores de personas de 65 y más años con discapacidad en la persistencia de un modelo de cuidado familiar. Rev Esp Geriatr Gerontol. 2018;53(2):66-72

2. González-Juárez L, Noreña-Peña AL, Cibanal-Juan L. Experiencia de inmigración de trabajadoras latinoamericanas en Alicante, España: un estudio etnográfico. Rev Lat Am Enfermagem 2014:22(5):857-65. doi: 10.1590 / 0104-1169.3559.2490

3. Gimeno-Feliu LA, Calderón-Larrañaga A, Díaz E, Laguna-Berna C Poblador-Plou B, Coscollar-Santaliestra C, et al. The definition of immigrant status matters: impact of nationality, country of origin, and length of stay in host country on mortality estimates. BMC Public Health. 2019;19:247. doi: 10.1186/s12889-019-6555-1

4. Villalobos-Dintrans P. Envejecimiento y cuidados a largo plazo en Chile: desafíos en el contexto de la OCDE. Rev Panam Salud Publica. 2017;41:86. doi: 10.26633/RPSP.2017.86
5. Díaz, M, Llorente M, Dema S. Tratamiento económico y modelización del cuidado. Convergencia. Rev Cienc Social [Internet]. 2018 [citado 19 de diciembre de 2018];25(76):37-56. Disponible en: http://www.scielo.org.mx/scielo.php?script=sci_arttext\&pi$d=\$ 1405-14352018000100037$

6. Glaser BG, Strauss AL. The discovery of grounded theory: Strategies for qualitative research. New Brunswick and London: Transaction Publishers [Internet]. 2009 [citado 23 de marzo de 2018]. Disponible en: http://www.sxf.uevora.pt/wp-content/ uploads/2013/03/Glaser_1967.pdf

7. Farmer T, Robinson K, Elliott SJ, Eyles J. Developing and implementing a triangulation protocol for qualitative health research Qual Health Res. 2006;16:377-94

8. Varela A. The perverse trinity from which Central American women flee: Femicide, state and market violence Debate Feminista. 2017;53:1-17. Disponible en: www.sciencedirect.com
9. Palomo MTM, González E0, Terrón JMM. Challenges in the professionalization of care work in Andalusia. Österreichische Zeitschrift für Soziologie. 2018;43(1):31-4.7

10. Oliva PL, Ordóñez L, Peinado P. Análisis de género desde la enfermería en cuidadoras inmigrantes de personas mayores. Ene. [Internet] 2017 [citado 23 de marzo de 2018];11(1). Disponible en: http://scielo.isciii.es/ scielo.php?script=sci_arttext\&pid=S1988-348X2017000100008\&lng=es

11. Pérez A, López S. Desigualdades a flor de piel: cadenas globales de cuidados. Concreciones en el empleo de hogar y políticas públicas. [monografía en Internet]. Madrid: ONU Mujeres. 2016 [citado 23 de marzo de 2018]:1-224. Disponible en: http://riberdis.cedd.net/ bitstream/handle/11181/5044/Desigualdades_a flor_de_piel. pdf? sequence=1\&rd=0031199660260311

12. Salami B, Duggleby W, Rajani F. The perspective of employers/ families and care recipients of migrant live-in caregivers: a scoping review. Health Soc Care Community. 2017;25(6):1667-78. 
13. Contel JC. Integrated care and the challenge of chronic illness. Enferm Clin. 2018:28(1):1-4

14. Yang FM, Kao ST. Commentary on "Rehabilitation Outcomes Associated With Foreign Domestic Workers as Caregivers". Arch Phys Med Rehabil. 2017;98(9):1903-5.

15. Fuentes V, Agrela B. Circuitos de precariedad de las cuidadoras bolivianas en España: Implicaciones familiares y supervivencias transnacionales. Int Migr [Internet]. 2018 [citado 11 de noviembre de 2018];9(3):121-44. Disponible en: <http://www.scielo.org.mx/scielo. php?script=sci_arttext\&pid=S1665-89062018000100121\&lng=es\&nrm=iso>. ISSN 1665-8906. http://dx.doi.org/10.17428/rmi.v9i34.348
16. Sterud T, Tynes T, Sivesind Mehlum I, Veiersted KV, Bergbom B, Aairila A, et al. A systematic review of working conditions and occupational health among immigrants in Europe and Canada. BMC Public Health. 2018;18:770. doi: 10.1186/s12889-018-5703-3

17. Moreno 0, Cardemil E. The role of religious attendance on mental health among Mexican populations: A contribution toward the discussion of the immigrant health paradox. Am J Orthopsychiatry. 2018;88(1):10-5. 2016;19. doi: 10.1037/ ort0000214

18. Bover A, Taltavull JM, Gastaldo D, Luengo R, Izquierdo MD, Juando-Prats C, et al. Calidad de vida de trabajadoras inmigran- tes latinoamericanas como cuidadoras en España. Gac Sanit. 2015;29(2):123-6

19. Jurado D, Alarcón RD, Martínez JM, Mendieta Y, Gutiérrez L, Gurpegui M. Factors associated with psychological distress or common mental disorders in migrant populations across the world. Rev Psiquiatr Salud Ment. 2017;10(1):45-58. doi:10.1016/i. rpsm.2016.04.004

20. Stepanikova I, Oates G. Perceived Discrimination and Privilege in Health Care: The Role of Socioeconomic Status and Race. Am J Prev Med. 2017;52(1):86-94. doi: 10.1016/j.amepre.2016.09.024 